\title{
Nosocomial Native Valve Endocarditis due to Methicillin-Susceptible Staphylococcus aureus in a Patient with Psoriatic Arthritis
}

\author{
HARUNA MIDORI KUMAMOTO* AND KENICHIRO YAITA** \\ * Department of General Internal Medicine, \\ ** Division of Infectious Diseases, Chidoribashi General Hospital, Fukuoka 812-8633, Japan \\ Received 7 July 2019, Accepted 15 October 2019 \\ J-STAGE advance publication 21 September 2021
}

Edited by YOSHIHIRO FUKUMOTO

\begin{abstract}
Summary: Nosocomial infective endocarditis is a relatively rare, but critical disease. A Japanese man in his $80 \mathrm{~s}$ with psoriatic arthritis that was being treated with prednisolone was admitted for dyspnea. The first diagnosis was healthcare-associated pneumonia, and piperacillin/tazobactam was started. The patient's blood culture was negative at the time of admission. During the treatment, acute kidney injury occurred due to the use of antibiotics. Hemodialysis was performed via a central venous catheter in the internal jugular vein. After treatment of pneumonia, the patient experienced a sudden onset of fever accompanied by a loss of consciousness. Blood cultures from the peripheral vein and the central venous catheter were positive for methicillin-susceptible Staphylococcus aureus. A transthoracic echocardiography revealed stringy strands of vegetation attached to the native mitral valve. Magnetic resonance imagings also showed a shower of emboli to the brain. Ceftriaxone and vancomycin were administered; however, the patient died following a massive cerebral infarction. Instances of in-hospital mortality from nosocomial endocarditis are higher than the rates of community-acquired endocarditis. Clinicians should pay close attention to risk factors for nosocomial infective endocarditis. These risk factors include long-term indwelling vascular devices, psoriatic arthritis and corticosteroid therapy.
\end{abstract}

Keywords nosocomial infective endocarditis, multiple cerebral infarcts, methicillin-susceptible Staphylococcus aureus, psoriatic arthritis

\section{INTRODUCTION}

A diagnosis of infective endocarditis (IE) remains challenging, and the mortality rate remains high. A recent report set the median time prior to a diagnosis of IE at 14 days [1]. Even with dramatic improvements, the hospital mortality rate is approximately $20 \%$ for these patients [2].

Furthermore, diagnosis and the management of nosocomial IE continues to be of heightened importance, because of mortality rates that are much higher than those of community-acquired IE [3-5]. The rea- son is that most of the patients are elderly and have comorbidities such as chemotherapy for malignancies, and hemodialysis [5].

Herein, we report a case of nosocomial IE due to methicillin-susceptible Staphylococcus aureus (MSSA). During the management of this case, multiple cerebral infarcts and a massive stroke occurred, which caused the death of the patient. We present this case and a review of the literature to discuss the clinical importance of recognizing nosocomial IE.

Corresponding Author: Kenichiro Yaita, M.D., Ph.D., Division of Infectious Diseases, Chidoribashi General Hospital, 5-18-1 Chiyo, Hakata-ku, Fukuokashi, Fukuoka 812-8633, Japan. Tel: +81-92-641-2761, E-mail: kyaita81@ gmail.com

Abbreviations: AKI, acute kidney injury; AML, anterior mitral leaflet; BUN, blood urea nitrogen; IE, infective endocarditis; MIC, minimum inhibitory concentrations; MRI, magnetic resonance imaging; MSSA, methicillin-susceptible Staphylococcus aureus; PIP/TAZ, piperacillin/tazobactam; PML, posterior mitral leaflet 


\section{CASE PRESENTATION}

A Japanese man in his 80s was referred to our hospital due to dyspnea and a wet cough that had continued for three days. The patient had a medical history of psoriatic arthritis, chronic heart failure (mild mitral valve regurgitation and mild tricuspid valve regurgitation), paroxysmal atrial fibrillation, chronic obstructive pulmonary disease, chronic kidney disease, hypertension, chronic pancreatitis, Alzheimer's disease and alcoholism. For the treatment of psoriatic arthritis, $15-20 \mathrm{mg} /$ day of prednisolone had been prescribed for at least 6 years. The patient's cigarette consumption was 62 pack-years, and his alcohol consumption was $100 \mathrm{~g} /$ day for approximately 60 years.

At the time of admission, the patient's vital signs were as follows: blood pressure, 195/97 $\mathrm{mmHg}$; heart rate, 117 beats/min; respiratory rate, 24 breaths $/ \mathrm{min}$; body temperature, $38.2^{\circ} \mathrm{C}$ and Glasgow Coma Scale, E4V5M6 (total 15/15). His height was $150.0 \mathrm{~cm}$ and his body weight was $44.9 \mathrm{~kg}$. Upon physical examination, coarse crackles were noted in his bilateral lung fields. No systolic murmur was pointed out. Scaly eruption on the whole body and edema on his extremities were shown. Laboratory data revealed low levels of plasma protein (total protein: $6.0 \mathrm{~g} / \mathrm{dL}$, albumin: 3.1 $\mathrm{g} / \mathrm{dL}$ ), a high level of inflammation (c-reactive protein: $6.39 \mathrm{mg} / \mathrm{dL}$, white blood cell count: $11,130 / \mu \mathrm{L}$ (neutrophil: $87.0 \%$ )), and chronic kidney disorder (serum creatinine $1.48 \mathrm{mg} / \mathrm{dL}$, blood urea nitrogen (BUN) $34.0 \mathrm{mg} / \mathrm{dL}$ ). The patient's chest X-ray films showed new ground glass opacity on the right lower lung field. Transthoracic echocardiography revealed very mild mitral valve regurgitation and inferior base hypokinesis on the left ventricle. There was no thrombus in the heart. These findings agreed with the results of an echocardiography performed a year prior to this admission. Based on his symptoms and the results of laboratory tests and imaging, we diagnosed pneumonia. We started piperacillin/tazobactam at a dose of $2.25 \mathrm{gm}$ administered intravenously every six hours after drawing a blood culture and collecting a sputum culture. The sputum before the antibiotic administration was classified as P2 in Miller \& Jones' classification. The smear of sputum had a polymicrobial pattern (Geckler's classification: group 3), and the results of the blood cultures on the day of admission were negative for bacteria.

On the 3rd hospital day, the patient experienced acute kidney injury (serum creatinine $3.28 \mathrm{mg} / \mathrm{dL}$, BUN $39.7 \mathrm{mg} / \mathrm{dL}$ ). Autoantibodies related to renal disorder were negative (proteinase-3-antineutrophil cytoplasmic antibody $<1.0 \mathrm{U} / \mathrm{mL}$, myeloperoxidaseanti-neutrophil cytoplasmic antibody $<1.0 \mathrm{U} / \mathrm{mL}$, antinuclear antibody $<40$ and anti-glomerular basement membrane antibody $<2.0 \mathrm{U} / \mathrm{mL}$ ), and hypocomplementemia was not detected (C3 $93 \mathrm{mg} / \mathrm{dL}, \mathrm{C} 429 \mathrm{mg} /$ $\mathrm{dL}$, and CH50 $32.7 \mathrm{U} / \mathrm{mL}$ ). We concluded that the acute kidney injury might have been caused by piperacillin/tazobactam. On the 4th hospital day, the regimen of piperacillin/tazobactam was changed to ceftriaxone. A further exacerbation of renal function (serum creatinine $4.66 \mathrm{mg} / \mathrm{dL}, \mathrm{BUN} 46.2 \mathrm{mg} / \mathrm{dL}$ ), occurred on the 5th hospital day and prompted the introduction of hemodialysis via a central venous catheter in the right internal jugular vein. Ceftriaxone was stopped on the 15th hospital day. The blood tests on the 22 nd hospital day showed c-reactive protein: $1.69 \mathrm{mg} / \mathrm{dL}$, white blood cell count: $10,330 / \mu \mathrm{L}$.

On the 26th day, a new onset of sepsis was documented. The patient's vital signs were as follows: blood pressure, 108/66 mmHg; heart rate, 120 beats/ min; body temperature, $40.2^{\circ} \mathrm{C}$, Glasgow Coma Scale E2V1M4 (total 6/15) and SpO2 95\% (room air). Laboratory data again revealed high levels of inflammation (c-reactive protein: $34.33 \mathrm{mg} / \mathrm{dL}$, white blood cell count: $22,800 / \mu \mathrm{L}$ (neutrophil: $95.6 \%$ )). After drawing blood cultures, new antibiotics, doripenem, was administered intravenously at a dose of $0.25 \mathrm{~g}$ every eight hours, and vancomycin was administered intravenously in a target trough concentration of approximately $15 \mu \mathrm{g} / \mathrm{mL}$. The intravenous central venous catheter was then removed in the suspicion of catheter-related bloodstream infection. A new central venous catheter was settled in the left internal jugular vein.

On the 28th hospital day, the blood culture and catheter-tip culture became positive for gram-positive cocci and a new small erythematous macular lesion was detected on the patient's left hand. Systolic murmur was not detected. During the work-up for IE on the 29th hospital day, a transthoracic echocardiography revealed stringy vegetations $(27 \mathrm{~mm}$ on the anterior mitral leaflet and $25 \mathrm{~mm}$ on the posterior mitral leaflet) (Figure 1a,b: arrows) and severe mitral valve regurgitation on the patient's native mitral valve. Moreover, diffusion weighted magnetic resonance imagings showed acute multiple infarcts on the bilateral cerebral hemispheres (Figure 2a). On the 30th hospital day, gram-positive cocci were identified as MSSA. Susceptibilities and minimum inhibitory concentrations (MIC) of this strain were as follows: ampicillin, susceptible $\quad(\mathrm{MIC} \leqq 0.25)$; cefazolin, susceptible $(\mathrm{MIC} \leqq 8)$; ceftriaxone, susceptible $(\mathrm{MIC} \leqq 8)$; cipro- 
floxacin, resistant (MIC $\geqq 4)$; and, vancomycin, susceptible $(\mathrm{MIC}=1)$. Finally, we arrived at a diagnosis of nosocomial IE with multiple cerebral infarcts due to MSSA. After drawing a follow-up blood culture, we changed the doripenem to ceftriaxone at a dose of $2 \mathrm{~g}$ administered intravenously every 12 hours in addition to vancomycin. The patient's consciousness level had not improved from Glasgow Coma Scale E2V1M4 (total 6/15), and the patient needed adrenaline administered at $0.05 \mu \mathrm{g} / \mathrm{kg} / \mathrm{min}$ for the shock status.

On the 37th hospital day, diffusion weighted magnetic resonance imagings confirmed an acute massive infarction of the left cerebral hemisphere (Figure 2b). Although the blood culture became negative within four days, and the vegetations and regurgitation on the mitral valve had improved (single stringy vegetation $(13 \mathrm{~mm})$ was only detected at this point) on the 38th hospital day, ultimately the patient died on the 45th hospital day. A postmortem was not performed. The clinical course is described in Figure 3.

\section{DISCUSSION}

This case report highlights a fatal case due to nosocomial IE caused by MSSA. The most common source of healthcare-associated IE is a vascular catheter $(48 \%)$, particularly hemodialysis and peripheral vein catheters [4]. Our case had a hemodialysis catheter as a possible entry site of bacteria. Active psoriatic arthritis [6] and long-term corticosteroid therapy [7] have also been documented as the risk factors for bacteremia. In addition, staphylococcal infection is common in the patients with psoriasis $[8,9]$. S. aureus could easily colonize on the skin of patients with erythroderma [8]. Recently a relationship between psoriasis and IE [10] has also been reported. We were able to locate only five reports of IE cases with psoriasis published in English, and three were due to $S$. $a u$ reus [8,11-14], which agrees with the facts of our case. Some combination of risk factors contributed to the occurrence of staphylococcal bacteremia and IE in our case.

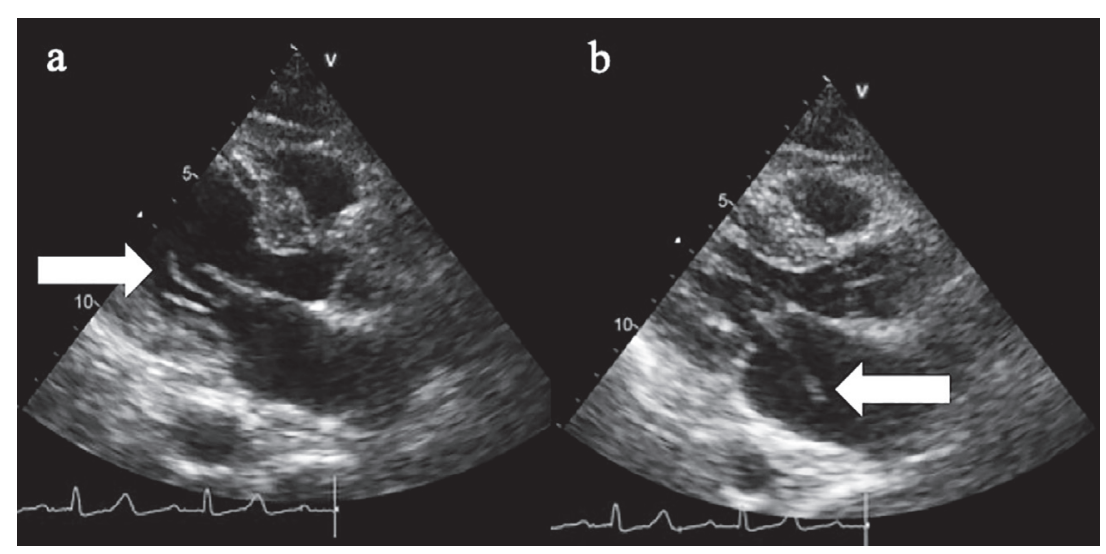

Fig. 1. Echocardiography revealed stringy vegetation (a, b. arrows) attached to the native mitral valve.

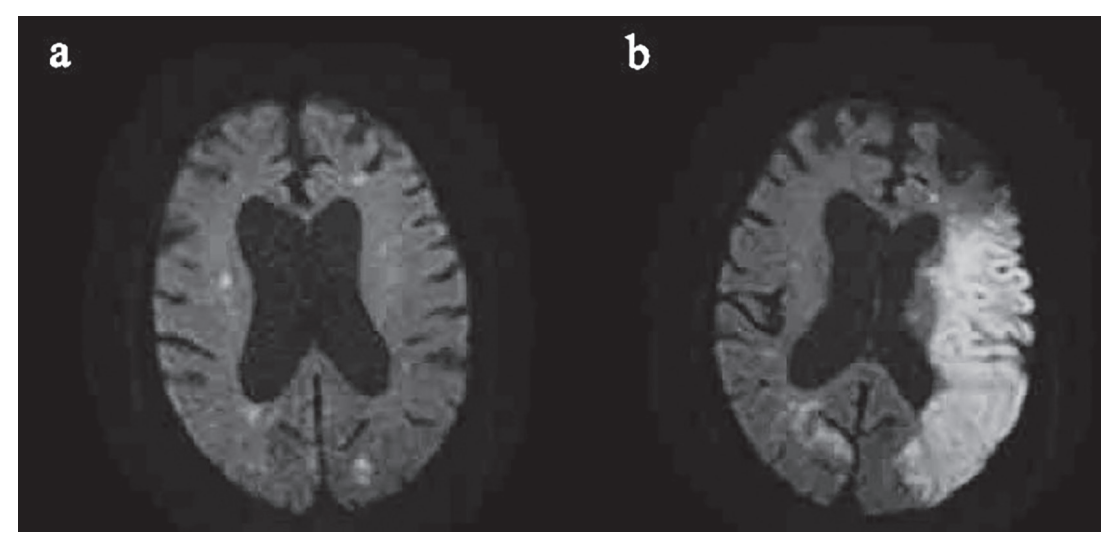

Fig. 2. Diffusion weighted magnetic resonance imagings confirmed shower emboli to the brain (a) followed by a massive infarction (b). 
MSSA that was the invading pathogen in our case was not a drug-resistant organism. Nosocomial IE due to MSSA, however, should not be underestimated. The neurologic complication rate (29\%) [15] and the rate of in-hospital mortality (22.4\%) [16] from IE due to $S$. aureus is higher than IE that is caused by other pathogens. Moreover, the rate of mortality from nosocomial IE is higher than that from community-acquired IE (39.5\% vs $18.6 \%)$ [17]. Such a high rate of mortality in nosocomial IE is caused by a patient population that tends to be elderly with comorbidities $[4,5]$, or by patients undergoing chemotherapy for malignancies [5]. Our case also had risk factors, elderly and the pathogen, of the in-hospital stroke and death.

Recent studies have reported that the differences in anti-staphylococcal drugs could influence the death rate $[18,19]$. In a retrospective study, empirical treatment by third-generation cephalosporins or beta-lactam/beta-lactamase inhibitors was associated with a higher rate of mortality compared with therapy using oxacillin/cefazolin [18]. On the other hand, vancomycin as the definitive therapy for MSSA bacteremia has also been related to a higher rate of mortality than when beta-lactams are used [19]. The American Heart Association guidelines were published in 2015 [20] and recommended treatment with nafcillin, oxacillin or cefazolin for the native valve endocarditis due to drug-susceptible staphylococci. Since we could not rule out meningitis without performing a spinal puncture, cefazolin was not selected. In addition, anti- staphylococcal penicillin was not available as a single agent in Japan. Given the complicated situation described above, we selected dual antibiotics as the definitive therapy in this case.

In addition to antibiotics, surgical valve replacement is also an important option for the treatment of infective endocarditis. The prevalence of the early valve replacement currently is increasing [21], and brain stroke is not a contraindication for the surgical treatment [21]. In our case, the operation was considered by the attending team; but was not performed finally due to the patient's severe neurologic damage [21] and shock status.

In conclusion, even with proper infectious disease consultation, and use of the appropriate antibiotics, nosocomial IE is nonetheless a critical disease. Clinicians must remember that indwelling vascular devices, psoriatic arthritis, and long-term corticosteroid therapies could cause not only uncomplicated bacteremia, but also fatal nosocomial IE.

ETHICS: The publication of this case report was approved by the Chidoribashi General Hospital Ethics Committee. (approved number $\mathrm{CH}-2019-04$ )

CONFLICT OF INTEREST: There are no conflicts of interest in connection with this article.

\section{ACKNOWLEDGEMENTS: None.}

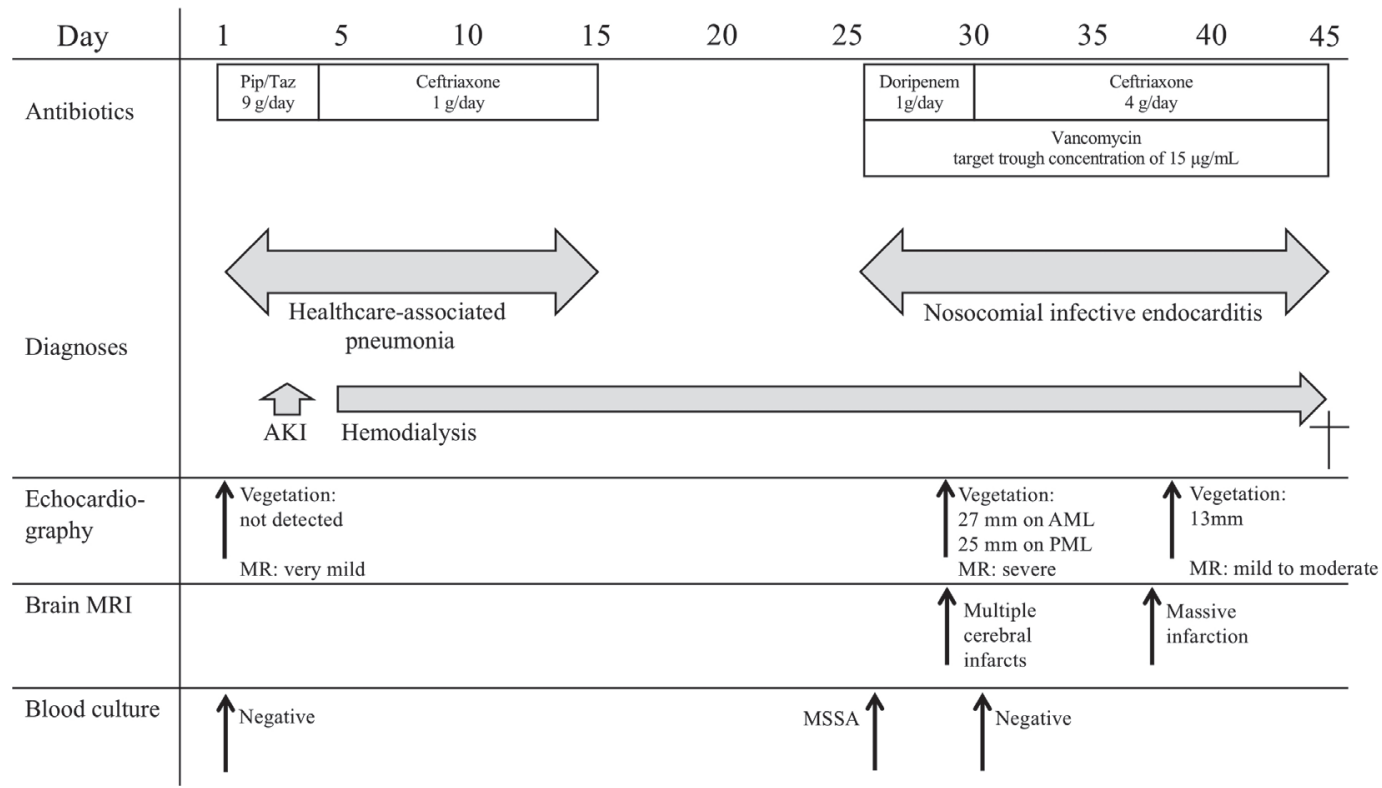

Fig. 3. Clinical course. AKI, acute kidney injury; AML, anterior mitral leaflet; MSSA, methicillin-susceptible Staphylococcus aureus; PIP/TAZ, piperacillin/tazobactam; PML, posterior mitral leaflet; 


\section{REFERENCES}

1. Fukuchi T, Iwata K, and Ohji G. Failure of Erly Diagnosis of Infective Endocarditis in Japan--a Retrospective Descriptive Analysis. Medicine 2014; 93:e237.

2. Slipczuk L, Codolosa JN, Davila CD, Romero-Corral A, Yun J et al. Infective Endocarditis Epidemiology Over Five Decades: A Systematic Review. PLoS One 2013; 8:e82665.

3. Martín-Dávila P, Fortún J, Navas E, Cobo J, Jiménez-Mena $\mathrm{M}$ et al. Nosocomial Endocarditis in a Tertiary Hospital: An Increasing Trend in Native Valve Cases. Chest. 2005; 128:772-779.

4. Lomas JM, Martínez-Marcos FJ, Plata A, Ivanova R, Galvez $\mathrm{J}$ et al. Healthcare-associated infective endocarditis: an undesirable effect of healthcare universalization. Clin Microbiol Infect. 2010; 16:1683-1690.

5. Hwang JW, Park SW, Cho EJ, Lee GY, Kim EK et al. Risk factors for poor prognosis in nosocomial infective endocarditis. Korean J Intern Med. 2018; 33:102-112.

6. Karmacharya P, Crowson CS, Poudel D, Shrestha P, and Wright K. Hospitalizations for Serious Infections in Psoriatic Arthritis Patients: Data from the National Inpatient Sample 2000-2014 [abstract]. Arthritis Rheumatol 2018; 70 (suppl 10).

7. Smit J, Kaasch AJ, Søgaard M, Thomsen RW, Nielsen H et al. Use of Glucocorticoids and Risk of CommunityAcquired Staphylococcus aureus Bacteremia: A Population-Based Case-Control Study. Mayo Clin Proc 2016; 91:873-880.

8. Green MS, Prystowsky JH, Cohen SR, Cohen JI, and Lebwohl MG. Infectious complications of erythrodermic psoriasis. J Am Acad Dermatol 1996; 34:911-914.

9. Hsu DY, Gordon K, and Silverberg JI. Serious infections in hospitalized patients with psoriasis in the United States. J Am Acad Dermatol 2016; 75:287-296.

10. Lauridsen TK, Bruun LE, Dahl A, Ahlehoff O, TorpPedersen $\mathrm{C}$ et al. Risk assessment of infective endocarditis in psoriasis patients, impact of gender and disease severity: A cohort study from Danish nationwide registries. 25th European Congress of Clinical Microbiology \& Infectious Diseases, Copenhagen, 2015.

11. Ostlere LS, Akhras F, Langtry JAA, and Staughton RCD. Generalized pustular psoriasis associated with bacterial endocarditis of the anterior papillary muscle. $\mathrm{Br} \mathrm{J}$ Dermatol. 1992; 127:187-188.

12. Wilkinson NM. Fatal bacterial endocarditis following aortic valve replacement in a patient being treated with methotrexate. J Heart Valve Dis 1999; 8:591-592.

13. Haque Hussain SS, Wallace M, Belham M, Rusk R, Carmichael AJ et al. Infective endocarditis complicating adalimumab therapy for psoriasis. Clin Exp Dermatol 2014; 39:555-556.

14. Mizuno T, Kiyosawa J, Fukuda A, Watanabe S, Kurose N et al. Infective endocarditis following tumor necrosis factor- $\alpha$ antagonist therapy for management of psoriatic erythroderma: a case report. J Med Case Rep. 2017; 11:35.

15. Heiro M, Nikoskelainen J, Engblom E, Kotilainen E, Marttila R et al. Neurologic Manifestations of Infective Endocarditis: A 17-Year Experience in a Teaching Hospital in Finland. Arch Intern Med 2000; 160:2781-2787.

16. Fowler VG Jr, Miro JM, Hoen B, Cabell CH, Abrutyn E et al. Staphylococcus aureus Endocarditis: A Consequence of Medical Progress. JAMA 2005; 293:3012-3021.

17. Giannitsioti E, Skiadas I, Antoniadou A, Tsiodras S, Kanavos K et al. Nosocomial vs. community-acquired infective endocarditis in Greece: changing epidemiological profile and mortality risk. Clin Microbiol Infect 2007; 13:763-769.

18. Paul M, Zemer-Wassercug N, Talker O, Lishtzinsky Y, Lev $\mathrm{B}$ et al. Are all beta-lactams similarly effective in the treatment of methicillin-sensitive Staphylococcus aureus bacteraemia? Clin Microbiol Infect 2011; 17:1581-1586.

19. McDanel JS, Perencevich EN, Diekema DJ, Herwaldt LA, Smith TC et al. Comparative Effectiveness of BetaLactams Versus Vancomycin for Treatment of MethicillinSusceptible Staphylococcus aureus Bloodstream Infections Among 122 Hospitals. Clin Infect Dis 2015; 61:361-367.

20. Baddour LM, Wilson WR, Bayer AS, Fowler VG Jr, Tleyjeh IM et al. Infective Endocarditis in Adults: Diagnosis, Antimicrobial Therapy, and Management of Complications: A Scientific Statement for Healthcare Professionals From the American Heart Association. Circulation 2015; 132:1435-1486.

21. Hoen B and Duval X. Clinical Practice. Infective endocarditis. N Engl J Med 2013; 368:1425-1433. 